\title{
Líneas de transmisión: su utilización en la interpretación de las medidas de impedancia en los estudios de corrosión ${ }^{(\cdot)}$
}

\author{
José Luis Polo Sanz" y José María Bastidas Rull**
}

\begin{abstract}
Resumen Se hace una revisión de las principales líneas de transmisión utilizadas en los estudios de corrosión. Se describen los electrodos constituidos por poros con formas cilíndrica e irregular y los electrodos coplanares. Finalmente, se comentan topologías de líneas de transmisión que tienen comportamiento de fase constante con la frecuencia.
\end{abstract}

Palabras clave Corrosión. Impedancia. Línea de transmisión. Electrodo poroso. Electrodo coplanar. Elemento de fase constante.

\section{Interpretation of corrosion impedance data using transmission lines}

\begin{abstract}
The transmission lines most frequently used in corrosion studies are described. Electrodes constituted by cylindrical pores and with irregular shapes are discussed. Coplanar electrodes are also studied. Finally, topologies of networks with fractional power frequency dependence are commented.
\end{abstract}

Keywords Corrosion. Impedance. Transmission line. Porous electrode. Coplanar electrode. Constant phase element.

\section{INTRODUCCIÓN}

En general, un circuito eléctrico equivalente que contenga un número finito de resistencias, bobinas y condensadores podría no modelar, con la aproximación deseada, las medidas experimentales de impedancia. Por ello, en la literatura se ha propuesto la utilización de elementos eléctricos distribuidos, definidos por funciones empíricas de impedancia. Por ejemplo, el circuito eléctrico indicado en la figura 1 incluye la impedancia de Warburg $\left(Z_{W}\right)^{[1]}$, que es un elemento eléctrico distribuido descrito por la expresión siguiente:

$$
\mathrm{Z}_{\mathrm{w}}=\sqrt{2} \frac{\sigma_{\mathrm{w}}}{\sqrt{\mathrm{j} \omega}}
$$

donde $\sigma_{w}$ es el coeficiente de Warburg, $\omega$ es la frecuencia angular y $\mathrm{j}^{2}=(-1)$. Obsérvese que, en la ecuación (1), la expresión compleja $1 / \sqrt{\mathrm{j}}$ puede escribirse como $(1-\mathrm{j}) / \sqrt{2} \mathrm{y}$, por tanto, es posible obtener una expresión más estandarizada de la impe-

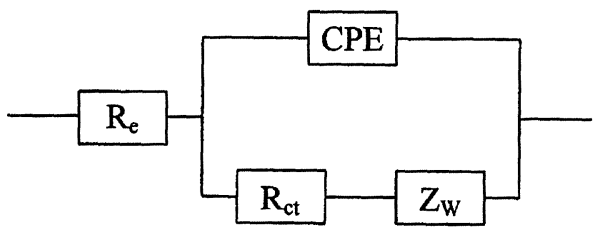

Figura 1. Circuito eléctrico equivalente con dos elementos distribuidos $C P E$ y $Z_{w}$.

Figure 1. Equivalent electrical circuit with two distributed elements $C P E$ and $Z_{w}$.

dancia de Warburg: $Z_{\mathrm{w}}=\left(\sigma_{\mathrm{w}} / \sqrt{\omega}\right)(1-\mathrm{j})$. Los otros parámetros de la figura 1 son la resistencia del electrólito $\left(\mathrm{R}_{\mathrm{e}}\right)$, el elemento de fase constante, CPE (constant phase element) y la resistencia de transferencia de carga $\left(\mathrm{R}_{\mathrm{ct}}\right)$.

La impedancia de Warburg, ecuación (1), involucra un proceso de difusión unidimensional a través de una capa de espesor infinito y ello no corresponde a una situación física real. LLopis y Colom ${ }^{[2]}$ introdujeron el concepto de capa de

(•) Trabajo recibido el día 21 de marzo de 2000 y aceptado en su forma final el día 20 de octubre de 2000.

$(*)$ E.U. Ingeniería Técnica Industrial. Universidad Castilla-La Mancha. Avda- Carlos III, s/n. 45071 Toledo (España).

$(* *)$ Centro Nacional de Investigaciones Metalúrgicas (CSIC). Avda. Gregorio del Amo, 8. 28040 Madrid (España). 
difusión de espesor finito, por lo que la expresión que modela la impedancia de un proceso de difusión $\left(Z_{D}\right)$ unidimensional en una capa de espesor finito es el elemento eléctrico distribuido, dado por la expresión siguiente:

$$
\mathrm{Z}_{\mathrm{D}}=\frac{1}{\sqrt{2}} \frac{\sigma_{\mathrm{w}}}{\sqrt{\mathrm{j} \omega}} \tanh \left(\sqrt{\mathrm{j} \omega \tau_{\mathrm{D}}}\right)
$$

donde $\tau_{\mathrm{D}}=\delta^{2} / \mathrm{D}$ es la constante de tiempo del proceso de difusión, siendo $\delta$ el espesor de la capa de difusión y $\mathrm{D}$ el coeficiente de difusión.

Por otro lado, el CPE de la figura 1 es otro elemento eléctrico distribuido que está definido por la función empírica de admitancia de la expresión siguiente:

$$
Y=Y_{p}(j \omega)^{\alpha}
$$

donde $\mathrm{Y}_{\mathrm{p}}$ es una constante independiente de la frecuencia. El exponente $\alpha$ es tal que $0<\alpha<1$ y su significado físico no está claro ${ }^{[3]}$. Si $\alpha=0$ el CPE es una resistencia de valor $R=1 / Y_{p}$. Cuando $\alpha=1$ es un condensador de valor $C=Y_{p}$. Si $\alpha=(-1)$ es una bobina de inducción $L=1 / Y_{p}$. Finalmente, cuando $\alpha=0,5$ la ecuación (3) se puede escribir como: $\mathrm{Y}=\mathrm{Y}_{\mathrm{p}} \sqrt{\mathrm{j} \omega}$, describiendo la admitancia de Warburg $^{[4]}$. En este caso, la relación entre el parámetro $Y_{p}$, ecuación (3), y el coeficiente de Warburg $\left(\sigma_{w}\right)$, ecuación (1), está dada por: $\sigma_{\mathrm{w}}=1 /\left(\mathrm{Y}_{\mathrm{p}} \sqrt{2}\right)$.

La técnica de impedancia electroquímica, EIS (electrochemical impedance spectroscopy), tiene su origen en la ingeniería eléctrica ${ }^{[5]}$. Por tanto, tiene interés utilizar circuitos eléctricos formados, exclusivamente, por resistencias, bobinas y condensadores que no incluyan componentes eléctricos definidos por funciones empíricas de impedancia, como las del circuito de la figura 1 .

Es conocido que en los electrodos rugosos y porosos, en los electrodos coplanares, en los electrodos con una superficie mucho mayor que la del contraelectrodo y, en general, en los fenómenos de corrosión por resquicios ("crevice corrosion", en lengua inglesa) se produce una distribución no uniforme de la densidad corriente alterna sobre el electrodo. Este fenómeno ha motivado a investigadores a abordar el estudio de electrodos porosos mediante la técnica EIS.

El objetivo de este artículo es describir las líneas de transmisión más utilizadas en los estudios de corrosión. Se pretende hacer una exposición de los fundamentos teóricos de dichas líneas de transmisión y discutir su utilidad en la interpretación de las medidas de impedancia.

\section{MODELADO DE ELECTRODOS POROSOS}

\subsection{Modelado de un poro con forma cilíndrica}

La impedancia de un poro que tiene forma cilíndrica se puede modelar, de manera sencilla, mediante una línea de transmisión que contenga parámetros distribuidos, de modo que las impedancias estén uniformemente repartidas por unidad de longitud a lo largo del eje de simetría del poro (Fig. 2).

En la figura 2, $R_{e}$ es la resistencia del electrólito en el que se encuentra sumergido el material electródico, $Z_{\mathrm{i}}$ es la impedancia de la superficie del electrodo alrededor de la boca del poro, $Z_{\mathrm{m}}$ es un parámetro uniformemente distribuido a lo largo del eje de simetría del poro que modela la impedancia del material del electrodo alrededor del poro y $\mathrm{Z}$ es la impedancia de la interfase electrodoelectrólito a lo largo de la pared del poro. Hay que indicar que la admitancia se distribuye uniformemente por unidad de longitud del poro. $R_{s}$ es la resistencia del electrólito dentro del poro, $Z_{u}$ es la impedancia de la interfase electrodo-electrólito en la base del poro $y$, finalmente, $Z_{b}$ es la impedancia

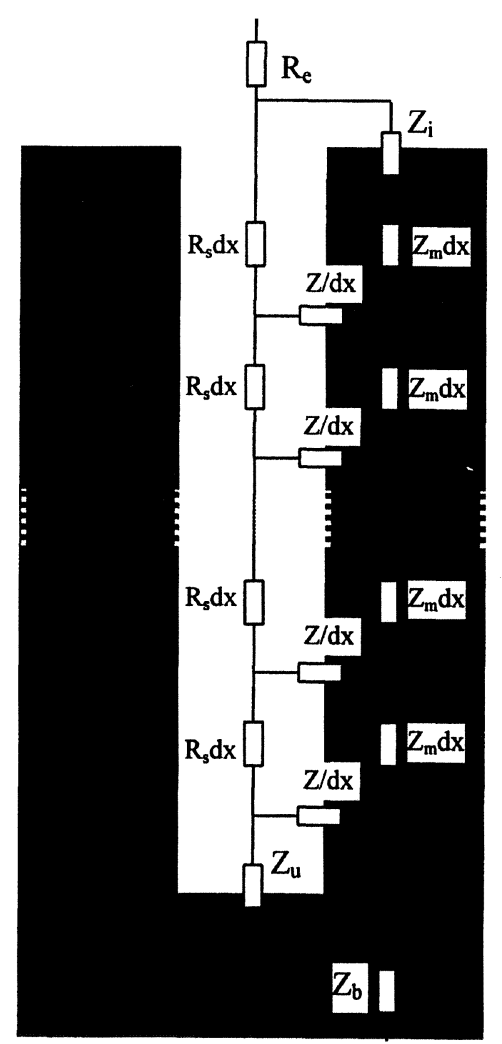

Figura 2. Circuito equivalente en línea de transmisión para la impedancia de un poro cilíndrico.

Figure 2. A transmission line as equivalent circuit for the impedance of a cylindrical pore.

Rev. Metal. Madrid 36 (2000) 
de la base del material. Los parámetros $R_{e}$ y $Z_{b}$ no forman parte de la impedancia del poro. Dichos parámetros se han incluido en la figura 2 para visualizar el circuito eléctrico de forma completa.

De Levie ${ }^{[6-9]}$ en una serie de artículos, clásicos en este tema, publicados en los años sesenta estudia la respuesta en frecuencia de electrodos porosos (poros cilíndricos) y rugosos (cavidades en forma de uve). Este autor propone un modelo basado en que los poros, llenos uniformemente de electrólito, tienen forma cilíndrica y la impedancia del material electródico es despreciable $\left(Z_{\mathrm{m}} \approx 0\right)$. Por otro lado, la superficie del electrodo alrededor de la boca del poro y la interfase electrodo-electrólito en la base del poro son aislantes, es decir, $Z_{\mathrm{i}} \rightarrow \infty$ y $\mathrm{Z}_{\mathrm{u}} \rightarrow \infty$ (Fig. 2).
Si, en el intervalo de frecuencias de interés, la longitud de penetración es muy pequeña frente a la longitud del poro ( $\mathrm{L}>3 \lambda$ éste se comporta como si tuviera longitud infinita. Por tanto, en la ecuación (4) se puede suponer que: $\operatorname{coth}\left(L \sqrt{R_{s} / Z}\right) \approx 1$, obteniendo la expresión siguiente:

$$
Z_{p}=\sqrt{R_{s} Z}
$$

Park y Macdonald ${ }^{[12]}$ proponen la utilización de la línea de transmisión finita propuesta por De Le$v^{\left[e^{[6]}\right.}$. Sin embargo, aquellos autores consideran la impedancia del material electródico definida por una resistencia $\left(Z_{m} \approx R_{m}\right)$ y un valor finito para el parámetro $Z_{\mathrm{u}}$. Es posible demostrar que $Z_{\mathrm{p}}$ está dada por la expresión siguiente:

$$
Z_{p}=\frac{R_{m} R_{s} L}{R_{m}+R_{s}}+\frac{2 R_{m} R_{s} \sqrt{\frac{R_{m}+R_{s}}{Z}}+\sqrt{\frac{R_{m}+R_{s}}{Z}}\left(R_{m}^{2}+R_{s}^{2}\right) \cosh \left(L \sqrt{\frac{R_{m}+R_{s}}{Z}}\right)+R_{s}^{2} \frac{R_{m}+R_{s}}{Z_{u}} \operatorname{senh}\left(L \sqrt{\frac{R_{m}+R_{s}}{Z}}\right)}{\left.\sqrt{\frac{R_{m}+R_{s}}{Z}\left(R_{m}+R_{s}\right.}\right)\left[\sqrt{\frac{R_{m}+R_{s}}{Z}} \operatorname{senh}\left(L \sqrt{\frac{R_{m}+R_{s}}{Z}}\right)+\frac{R_{m}+R_{s}}{Z_{u}} \cosh \left(L \sqrt{\frac{R_{m}+R_{s}}{Z}}\right)\right]}
$$

Es posible demostrar que en el modelo propuesto por De Levie la impedancia del poro $\left(Z_{p}\right)$, de longitud L (profundidad del poro), está dada por la expresión siguiente:

$$
Z_{p}=\sqrt{R_{s} Z} \operatorname{coth}\left(L \sqrt{\frac{R_{s}}{Z}}\right)
$$

Raistrick $^{[10]}$ y Ohmori et al. ${ }^{[11]}$ han utilizado la ecuación (4) para calcular la impedancia de un electrodo poroso. Para ello han considerado que la impedancia $(Z)$ de la interfase electrodo-electrólito a lo largo de la pared del poro es exclusivamente capacitiva

$$
Z=\frac{1}{j \omega C}
$$

Park y Macdonald ${ }^{[12]}$ consideran que el parámetro $Z$ está descrito por un condensador $\left(C_{w}\right)$ en paralelo con una resistencia $\left(R_{w}\right)$ que modelan, respectivamente, la capacidad y la resistencia de la interfase electrodo-electrólito a lo largo de la pared del poro.

Si se representa $Z_{p}$ en forma polar: $Z_{p}=\left|Z_{p}\right|_{\psi}^{[6]}$ es posible definir la longitud de penetración $(\lambda)$ mediante la expresión siguiente:

$$
\lambda=\frac{1}{\cos (\psi / 2)} \sqrt{\frac{|Z|}{R_{s}}}
$$

Obsérvese que, si se considera que la resistencia $\left(\mathrm{R}_{\mathrm{m}}\right)$ del material electródico es despreciable $\left(\mathrm{R}_{\mathrm{m}} \approx 0\right)$ y la interfase electrodo-electrólito en la base del poro es aislante $\left(Z_{\mathrm{u}} \rightarrow \infty\right)$, la impedancia de la ecuación (8) coincide con la impedancia del poro calculada por De Levie (ec. 4).

Ren y Pickup ${ }^{[13]}$ han modelado el transporte de materia mediante la utilización de dos líneas de transmisión en paralelo. Con este modelo, los autores consiguen una mejor simulación de los datos experimentales.

\subsection{Modelo de un poro no cilíndrico con forma irregular}

Considérese la situación más general de un poro no cilíndrico con forma irregular (Fig. 3). El esquema de la línea de transmisión que modela esta situación está indicado en la figura 4. Obsérvese que, debido a la geometría irregular, los parámetros no se encuentran uniformemente distribuidos como ocurría en la figura 2 , sino que dependen de la distancia $(\mathrm{x})$ a la boca del poro definiendo una línea de transmisión no uniforme ${ }^{[14]}$. Hay que indicar que salvo la dependencia señalada, los parámetros de la figura 4 tienen el significado de la figura 2 .

Eloot et al. ${ }^{[15]}$ proponen un método matricial para modelar un poro con forma irregular. Los autores dividen el poro en $\mathrm{N}$ discos. El espesor de cada disco se elige de manera que dicho disco pueda 


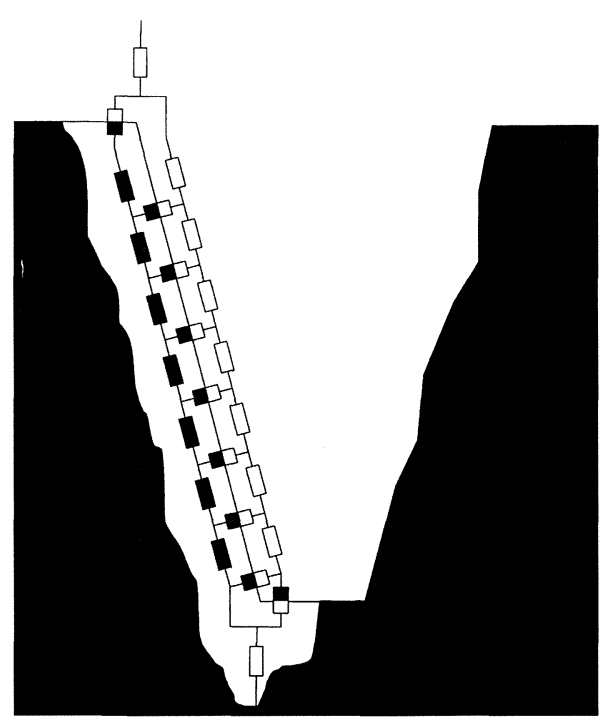

Figura 3. Circuito equivalente en línea de transmisión para la impedancia de un poro irregular. La impedancia del material no es despreciable.

Figure 3. A transmission line as equivalent circuit for the impedance of an irregular pore. The electrode material's impedance is not negligible.

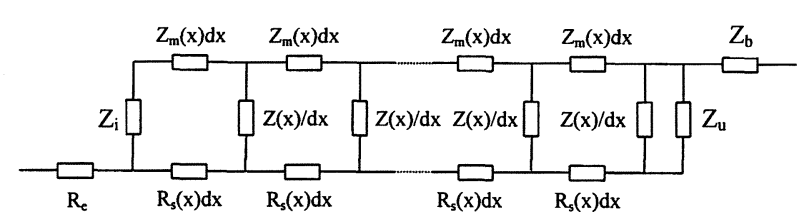

Figura 4. Línea de transmisión para el poro de la figura 3.

Figure 4. Transmission line for figure 3 pore.

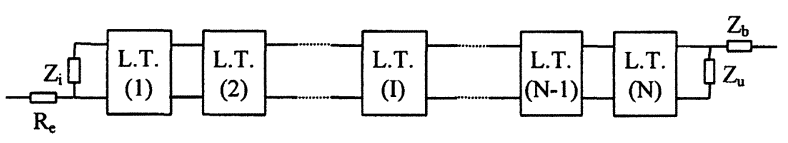

Figura 5. $\mathrm{N}$ líneas de transmisión dispuestas en serie.

Figure 5. $\mathrm{N}$ transmission lines connected in series.

donde $\alpha^{\prime}, \beta^{\prime}, \xi^{\prime}$ у $\eta^{\prime}$ son los elementos de la matriz siguiente:

$$
\left(\begin{array}{l}
\alpha^{\prime} \beta^{\prime} \\
\xi^{\prime} \eta^{\prime}
\end{array}\right)=\prod_{i=N}^{1}\left[\begin{array}{c}
1 \\
-\sqrt{\frac{1}{R_{s}^{(i)} Z^{(i)}}} \operatorname{tgh}\left(L_{i} \sqrt{\frac{R_{s}^{(i)}}{Z^{(i)}}}\right) \\
1
\end{array}\right]
$$

ser modelado, con suficiente aproximación, mediante una línea de transmisión de parámetros uniformemente distribuidos. Es decir, la línea de transmisión no uniforme que modela el poro con forma irregular se aproxima mediante $\mathrm{N}$ líneas de transmisión de parámetros uniformemente distribuidos y dispuestas consecutivamente (Fig. 5). Los parámetros de la línea de transmisión i-ésima, de longitud $\mathrm{L}_{\mathrm{i}}$, se anotan con el superíndice (i).

Eloot et al. ${ }^{[15]}$ calculan la impedancia del poro de la forma siguiente: si la impedancia del electrodo es despreciable frente a la resistencia del electrólito $\left(\mathrm{Z}_{\mathrm{m}}{ }^{(\mathrm{i})} \approx 0\right.$ con $\left.\mathrm{i}=1, \ldots, \mathrm{N}\right)$ y la superficie del electrodo alrededor de la boca del poro es aislante $\left(Z_{\mathrm{i}} \rightarrow \infty\right)$ se obtiene que la impedancia del poro $\left(\mathrm{Z}_{\mathrm{p}}\right)$ está dada por la expresión siguiente:

$$
Z_{p}=-\frac{x^{\prime}-Z_{u} a^{\prime}}{h^{\prime}-Z_{u} b^{\prime}}
$$

La ecuación (10) resulta de multiplicar las $\mathrm{N}$ matrices características correspondientes a cada una de las $\mathrm{N}$ líneas de transmisión que modelan el poro.

La expresión de la impedancia del poro $\left(Z_{p}\right)$, (ec. 9), se simplifica considerando que $Z_{u} \rightarrow \infty$ y se obtiene la expresión siguiente:

$$
Z_{p}=\lim _{Z_{u} \rightarrow \infty}\left(-\frac{\xi^{\prime}-Z_{u} \alpha^{\prime}}{\beta^{\prime}-Z_{u} \alpha^{\prime}}\right)=-\frac{\alpha^{\prime}}{\beta^{\prime}}
$$

Si se considera una única línea de transmisión, $\mathrm{i}=1$, la expresión de la impedancia de la ecuación (11) coincide con la de la ecuación (4), calculada por De Levie ${ }^{[6]}$.

Keiser et al. ${ }^{[16]}$ calculan la impedancia de poros con forma de sólidos de revolución. Estos autores dividen el poro en $\mathrm{N}$ discos de igual espesor. Cada 
disco lo modelan mediante un circuito $\mathrm{R}_{\mathrm{i}} \mathrm{C}_{\mathrm{i}}$, en el que $R_{i}$ simula la resistencia del electrólito dentro del disco i-ésimo y $\mathrm{C}_{\mathrm{i}}$ modela la capacidad de la doble capa electroquímica, formada entre el electrólito y la pared del poro en el disco i-ésimo considerado. Se supone que la impedancia del electrodo es despreciable y que la boca y la base del poro son aislantes. $\mathrm{Al}$ interconectar los circuitos $\mathrm{R}_{\mathrm{i}} \mathrm{C}_{\mathrm{i}}$ correspondientes a cada disco se obtiene una red en escalera, como la indicada en la figura 6.

Si $L$ es la profundidad del poro y $\mathrm{L} / \mathrm{N}$ es la anchura de cada disco, la impedancia global del poro se calcula mediante la fórmula recursiva siguien$\mathrm{te}^{[16]}$ :

$$
Z_{(i-1)}^{*}=\frac{1}{N\left(\frac{r_{i}}{r}\right)^{2}}+\frac{1}{j \frac{1}{2 N}\left(\frac{1}{\lambda_{1}}\right)^{2} \frac{r_{1}}{r}+\frac{1}{Z_{(i)}^{*}}}
$$

donde $Z_{(i)}^{*}$ es la impedancia normalizada del disco i-ésimo, obtenida al dividir la impedancia de ese disco por la resistencia del electrólito (con una resistividad $\rho_{\mathrm{s}}$ ) del poro completo, como si éste fuera cilíndrico y de radio medio $r, r_{i}$ es el radio medio del disco i-ésimo y, finalmente, $\lambda_{\mathrm{r}}$ es la longitud de penetración definida por la expresión: $\lambda_{\mathrm{r}}=\sqrt{\mathrm{r}} / 2 \sqrt{\rho_{\mathrm{s}} \omega \mathrm{C}_{\mathrm{dl}}}$, donde $\mathrm{C}_{\mathrm{dl}}$ es la capacidad de la doble capa electroquímica por unidad de superficie $^{[16-18]}$.

Debido a que se considera que la base del poro es aislante, el valor de comienzo de las iteraciones es $Z_{(\mathrm{N})}^{*}=\infty^{[16]}$.

\section{MODELADO DE ELECTRODOS COPLANARES}

Como se ha indicado anteriormente, otra situación en la que se puede producir una distribución no uniforme de la corriente alterna es en los denominados electrodos coplanares, constituidos por bandas rectangulares yuxtapuestas. Este tipo de electrodos, de diseño especial, es muy utilizado en los estudios de corrosión atmosférica y de metales pintados $^{[19-22]}$.

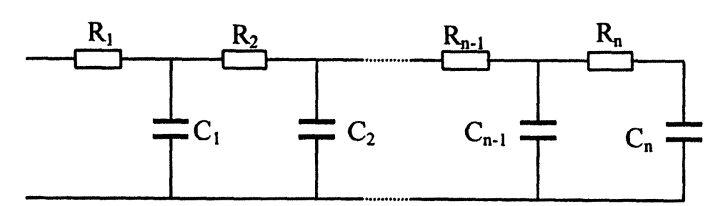

Figura 6. Red en escalera para el modelado de un poro irregular.

Figure 6. Ladder network to model an irregular pore.
Nishikata et al. ${ }^{[23}$ y 24] utilizan una línea de transmisión basada en las ecuaciones (4) y (7) para modelar la impedancia de electrodos coplanares cubiertos por capas de electrólito muy delgadas. La impedancia $(Z)$ se describe mediante un circuito R-CPE en paralelo y su valor está dado por la expresión siguiente:

$$
Z=\frac{R}{1+R Y_{p}(j \omega)^{a}}
$$

Para un valor de $\alpha=1$ en la ecuación (13), la ecuación (7) define la cuarta parte de una lemniscata en el plano complejo.

Feliu et al. ${ }^{[25]}$ proponen utilizar una línea de transmisión y, mediante una fórmula recursiva, realizan el cálculo numérico de la impedancia de electrodos coplanares rectangulares.

\section{MODELADO DEL COMPORTAMIENTO DE FASE CONSTANTE CON LA FRECUENCIA}

Eligiendo convenientemente los parámetros de las líneas de transmisión analizadas es posible obtener un comportamiento de fase constante con la frecuencia. A continuación se describen dos ejemplos.

\subsection{Modelado de la doble capa electroquímica}

$\mathrm{Liu}^{[26]}$ ha considerado un electrodo con una superficie fractal descrita por el conjunto de Cantor ${ }^{[27]}$ (ver figura 7). Cada gruta se modela mediante un circuito RC y se obtiene la línea de transmisión de la figura 7. En dicha figura se observa que la resistencia R (del electrólito dentro de la gruta) se incrementa por la razón $a$, en cada paso del circuito, debido a la reducción en la sección de la gruta. La capacidad (C) es constante en cada paso del circuito y modela la capacidad de la doble capa electroquímica dentro de la gruta.

La línea de transmisión de la figura 7 modela un CPE, en el que el exponente $(\alpha)$ está relacionado con la dimensión fractal $\left(D_{f}\right)$ de la superficie del electrodo (Fig. 7) ${ }^{[26]}$, mediante la expresión siguiente:

$$
\alpha=3-D_{\mathrm{f}}
$$

Wang y Bates ${ }^{[28]}$ demuestran que la impedancia que presentan algunos poros con formas especiales tiene un comportamiento de fase constante con la frecuencia. 


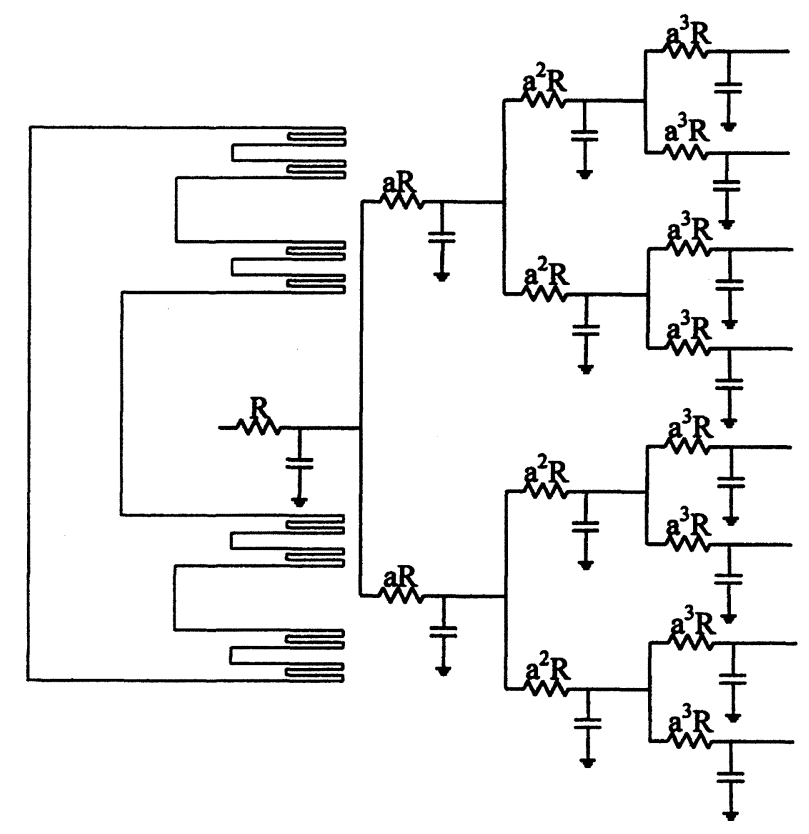

Figura 7. Superficie fractal descrita por el modelo de Cantor y línea de transmisión para modelar el comportamiento de fase constante (CPE).

Figure 7. Fractal surface described by the Cantor model, and transmission line to model the behaviour of a constant phase element (CPE).

\subsection{Modelado de los procesos de difusión}

Es necesario indicar que, aunque en el modelado de los procesos de difusión se utilicen las líneas de transmisión, explicadas anteriormente, y se conserve la notación de sus parámetros, el significado físico de éstos es distinto al definido en el Apartado 2.1. En el comportamiento de fase constante no se modela la impedancia de un poro, sino que se utiliza la línea de transmisión como una analogía del proceso de difusión para conseguir una representación matemática equivalente de la impedancia de dicho proceso.

Si se considera la línea de transmisión descrita por la ecuación (7), con una impedancia (Z) definida por la ecuación (5), resulta un comportamiento de fase constante de $45^{\circ}$, debido a que $\left|\arg \left(\sqrt{R_{s} / j \omega C}\right)\right|=45^{\circ}$. Esta línea de transmisión modela la impedancia de Warburg donde, por analogía con la ecuación (1), $\sigma_{\mathrm{w}}=\sqrt{2 \mathrm{R}_{\mathrm{s}} / \mathrm{C}}$.

Sin embargo, dicho ángulo de valor $45^{\circ}$ puede ser distinto al considerar ramificaciones de la propia línea de transmisión en otras nuevas líneas de transmisión, dependiendo el valor del ángulo de fase constante del grado de ramificación. Por ejemplo, supóngase que $Z$ esta descrita por otra línea de transmisión que también satisface la ecua- ción (7). En este caso, se obtiene un comportamiento de fase constante de $22,5^{\circ}$, debido a que $\left|\arg \left(\sqrt{\left.\mathrm{R}_{\mathrm{s} 1} \sqrt{\mathrm{R}_{\mathrm{s} 2} / \mathrm{j} \overline{\mathrm{j}}}\right)}\right)\right|=22,5^{\circ}$.

La impedancia de difusión de la ecuación (2) se puede modelar mediante la línea de transmisión de la figura 2 , considerando que $Z_{\mathrm{i}} \rightarrow \infty$ y $Z_{\mathrm{u}}=0$. La impedancia de la línea de transmisión $\left(Z_{\mathrm{lt}}\right)$ viene dada por la ecuación (9), en la que $Z_{\mathrm{u}}=0$. Los parámetros '̧' y $\eta^{\prime}$ se obtienen de la ecuación (10) con i=1. Finalmente, $Z_{\mathrm{lt}}$ está dada por la expresión siguiente:

$$
\mathrm{Z}_{\mathrm{lt}}=\sqrt{\mathrm{R}_{z} \mathrm{Z}} \operatorname{tgh}\left(\mathrm{L} \sqrt{\frac{\mathrm{R}_{\mathrm{s}}}{\mathrm{Z}}}\right)
$$

Mediante la ecuación (15) y considerando que la impedancia (Z) es capacitiva, la ecuación (5) se puede escribir de la forma siguiente:

$$
\mathrm{Z}_{\mathrm{lt}}=\sqrt{\frac{\mathrm{R}_{\mathrm{s}}}{\mathrm{C}}} \frac{1}{\sqrt{\mathrm{j} \omega}} \operatorname{tgh}\left(\sqrt{\mathrm{j} \omega \mathrm{R}_{\mathrm{s}} \mathrm{CL}^{2}}\right)
$$

La ecuación (16) tiene la misma expresión que la impedancia de difusión en una capa de espesor finito. Por analogía con la ecuación (2), se obtiene que: $\sigma_{w}=\sqrt{2 R_{s} / C}$ y $\tau_{D}=R_{s} C L^{2}$, donde $\delta=L$ y $\mathrm{D}=1 /\left(\mathrm{R}_{\mathrm{s}} \mathrm{C}\right)$.

Wang $^{[29]}$ analiza la posibilidad de utilizar una red en escalera, como la de la figura 6, propuesta por Schrama en un trabajo no publicado. Schrama, según Wang ${ }^{[29]}$, demuestra que eligiendo convenientemente el valor de los parámetros de la figura 6 , es posible conseguir que la red tenga un comportamiento de fase constante con la frecuencia y que modele el comportamiento de Warburg generalizado (ángulo de la "cola" de difusión distinto de $45^{\circ}$ ). La elección de dichos parámetros es de la forma siguiente:

$$
\begin{aligned}
& \mathrm{R}_{\mathrm{k}}=2 \mathrm{~h}^{\alpha} \frac{\Gamma(1-\alpha)}{\Gamma(\alpha)} \frac{\Gamma(\mathrm{k}+\alpha)}{\Gamma(\mathrm{k}+1-\alpha)}-\mathrm{h}^{\alpha} \delta_{\mathrm{ko}} \\
& \mathrm{C}_{\mathrm{k}}=(2 \mathrm{k}+1) \mathrm{h}^{1-\alpha} \frac{\Gamma(\alpha)}{\Gamma(1-\alpha)} \frac{\Gamma(\mathrm{k}+1-\alpha)}{\Gamma(\mathrm{k}+1+\alpha)}
\end{aligned}
$$

donde $R_{k}$ y $C_{k}$ son los parámetros del circuito de la figura $6, \Gamma$ es la función gamma, $\delta$ es la delta de Kronecker, $\mathrm{k}=0,1,2, . ., \alpha$ se ha definido anteriormente, $\mathrm{y} h$ es un número real positivo suficientemente pequeño. Para $\alpha>0,5$ cuando $k$ se incrementa, resulta que $R_{k}$ crece y $C_{k}$ decrece. Contrariamente, para $\alpha<0,5$ cuando $\mathrm{k}$ se 
incrementa, $R_{k}$ decrece y $C_{k}$ crece. Obsérvese que si $\alpha=0,5$ las resistencias y condensadores son siempre iguales entre sí, ya que no dependen de k. En ese caso se obtiene la impedancia de Warburg.

\section{ALGUNOS EJEMPLOS SENCILLOS}

La figura 8 muestra el diagrama de impedancia de un poro cilíndrico. La simulación se realizó utilizando la ecuación (8). Los parámetros de la simulación fueron los siguientes: $R_{m}=12 \mathrm{M} \Omega \mathrm{cm}$, $\mathrm{R}_{\mathrm{s}}=200 \mathrm{k} \Omega \mathrm{cm}$, y $\mathrm{L}=0,025 \mathrm{~cm}$. Obsérvese que tanto $\mathrm{R}_{\mathrm{m}}$ como $\mathrm{R}_{\mathrm{s}}$ son parámetros distribuidos por unidad de longitud de línea y, por tanto, sus unidades son $\mathrm{k} \Omega \mathrm{cm}$. Los parámetros $\mathrm{Z}_{\mathrm{y}} \mathrm{Z}_{\mathrm{u}}$ se han modelado mediante los circuitos $\mathrm{R}_{w} \mathrm{C}_{w}$ y $\mathrm{R}_{\mathrm{u}} \mathrm{C}_{\mathrm{u}}$ en paralelo, respectivamente, $R_{w}=30 \mathrm{k} \Omega \mathrm{cm}^{3}, \mathrm{C}_{\mathrm{w}}=700$ $\mu \mathrm{F} / \mathrm{cm}^{3}, \mathrm{R}_{\mathrm{u}}=280 \mathrm{k} \Omega \mathrm{cm}^{2}$ y $\mathrm{C}_{\mathrm{u}}=0,60 \mu \mathrm{F} / \mathrm{cm}^{2}$. Nótese que la inversa del parámetro $\mathrm{Z}$ (la admitancia) está distribuida por unidad de longitud de línea y, por tanto, las unidades de $Z$ son $\mathrm{k} \Omega \mathrm{cm}^{3}$ en vez de $\mathrm{k} \Omega \mathrm{cm}^{2}$. En consecuencia, $\mathrm{R}_{\mathrm{w}}$ y $\mathrm{C}_{\mathrm{w}}$ se expresan en $\mathrm{k} \Omega \mathrm{cm}^{3}$ y $\mu \mathrm{F} / \mathrm{cm}^{3}$, respectivamente. Por el contrario, $\mathrm{Z}_{\mathrm{u}}$ es un parámetro localizado y no distribuido, por lo que sus unidades son $\mathrm{k} \Omega \mathrm{cm}^{2}$. Es necesario indicar que los valores de los ejes de la figura 8 son del orden de $\mathrm{k} \Omega \mathrm{cm}^{2}$. Ello es debido a que dicha figura simula un sólo poro. En el caso de que el electrodo esté constituido por 10.000 poros y, considerando que la impedancia global del electrodo está definida por las impedancias correspondientes a cada uno de los poros en paralelo, los ejes del diagrama de la figura 8 se multiplicarían por 10.000, reduciéndose las unidades al orden de $\Omega \mathrm{cm}^{2}$.

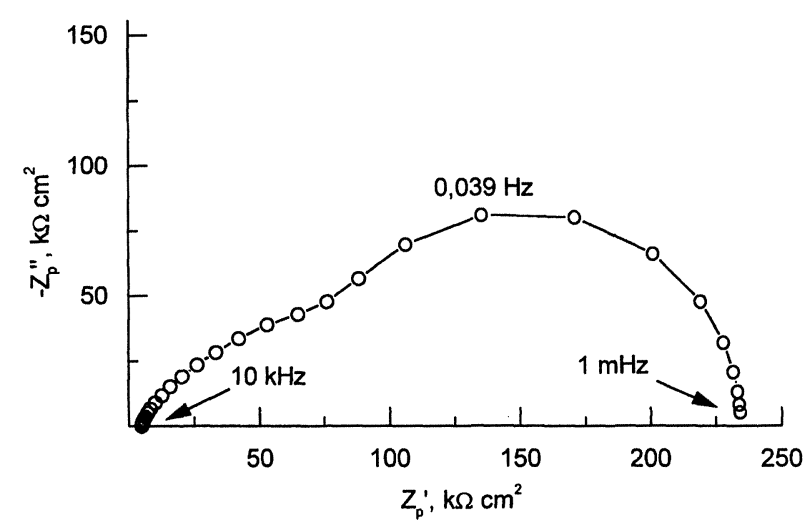

Figura 8. Diagrama de impedancia de un poro cilíndrico, ecuación (8).

Figure 8. Impedance diagram for a cylindrical pore, equation (8).
La figura 9 se ha obtenido de la misma forma que la figura 8 . Se ha considerado que la resistencia del material electródico es despreciable: $R_{m} \approx 0$, que la impedancia $(Z)$ de la interfase electrodo-electrólito a lo largo de la pared del poro es exclusivamente capacitiva: $\mathrm{C}_{\mathrm{w}}=700 \mu \mathrm{F} / \mathrm{cm}^{3}$, y que la base del poro es aislante: $Z_{u} \rightarrow \infty$. Con estas simplificaciones, dicha figura corresponde a la simulación de la impedancia del poro de la ecuación (4) o de la ecuación (11), en la que los parámetros $\alpha^{\prime}$ y $\beta^{\prime}$ se obtienen, a su vez, de la ecuación (10) para el caso particular de $\mathrm{i}=1$. En la figura 9 , se observa que en el intervalo de frecuencia de $10 \mathrm{kHz}$ a $39,8 \mathrm{~Hz}$ el poro se puede considerar de longitud semiinfinita, y está descrito por la ecuación (7). Este comportamiento se debe a que la longitud de penetración de la señal de corriente alterna es menor que la longitud del poro para frecuencias superiores a $39,8 \mathrm{~Hz}$, por tanto, el poro se puede considerar de longitud semiinfinita para ese intervalo de frecuencia.

La figura 10 muestra el diagrama de Nyquist de un proceso de difusión en una capa de espesor finito. Dicha figura se ha modelado utilizando la línea de transmisión de la figura 2, en la que se ha considerado que $Z_{\mathrm{i}} \rightarrow \infty$ y $Z_{\mathrm{u}}=0$. La simulación de la figura 10 se ha realizado mediante la ecuación (16). Los parámetros utilizados han sido $\mathrm{R}_{\mathrm{s}}=22,5 \mathrm{k} \Omega \mathrm{cm}, \mathrm{C}=4,5 \mathrm{~F} / \mathrm{cm}^{3}, \mathrm{y} \mathrm{L}=0,0063 \mathrm{~cm}$. Se

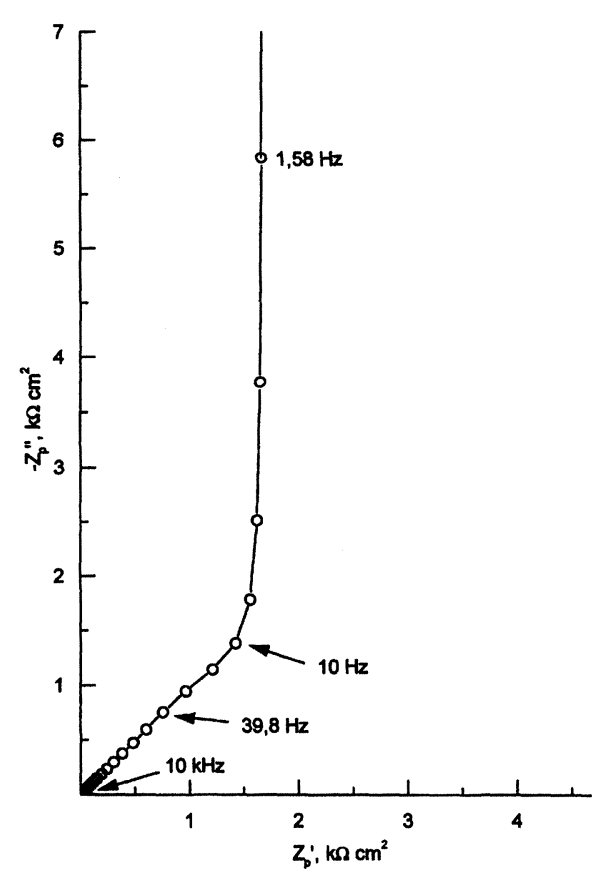

Figura 9. Diagrama de impedancia de un poro cilíndrico, ecuación (4).

Figure 9. Impedance diagram for a cylindrical pore, equation (4). 


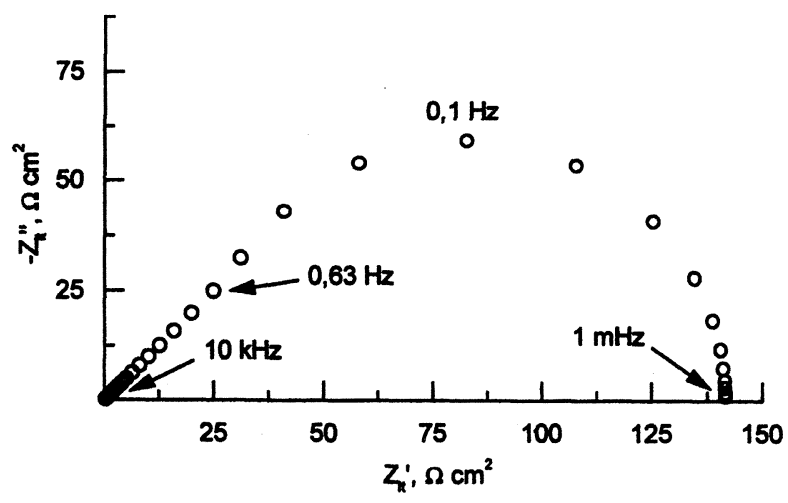

Figura 10. Diagrama de impedancia de un proceso de difusión modelado mediante una línea de transmisión, ecuación (16).

Figure 10. Impedance diagram for a diffusional process modelled using a transmission line, equation (16).

obtiene un coeficiente de Warburg: $\sigma_{\mathrm{w}}=\sqrt{2 \mathrm{R}_{\mathrm{s}} / \mathrm{C}}=$ $100 \Omega \mathrm{cm}^{2} / \mathrm{s}^{0,5}$ y una constante de tiempo del proceso de difusión: $\tau_{\mathrm{D}}=\mathrm{R}_{\mathrm{s}} \mathrm{CL}^{2}=4 \mathrm{~s}$. El coeficiente de difusión tiene el valor de: $D=1 /\left(R_{s} C\right)=10^{-5}$ $\mathrm{cm}^{2} / \mathrm{s}$. En la figura 10 se observa que en el intervalo de frecuencia de $10 \mathrm{kHz}$ a $0,63 \mathrm{~Hz}$ la impedancia está descrita por la ecuación (1). Esto se debe a que la señal de corriente alterna, en ese intervalo de frecuencia, no logra atravesar la capa de difusión y ésta se puede considerar como infinita para frecuencias superiores a $0,63 \mathrm{~Hz}$.

\section{CONCLUSIONES}

Se analizan las posibilidades de utilizar las líneas de transmisión en la interpretación de las medidas de impedancia. En particular, se analiza la impedancia de poros con forma cilíndrica, poros no cilíndricos con forma irregular y electrodos coplanares rectangulares. Asimismo, se han visto topologías de líneas de transmisión que poseen un comportamiento de fase constante con la frecuencia.

El método propuesto por De Levie es el más sencillo, debido a que este autor considera poros cilíndricos. El método propuesto por Eloot et al. es el más general para calcular la impedancia de un poro irregular. El poro se modela mediante $\mathrm{N}$ líneas de transmisión de parámetros uniformemente distribuidos dispuestas en serie y la impedancia se calcula mediante un método matricial. El procedimiento de Keiser et al. es una simplificación del procedimiento anterior, resultando una línea de transmisión de parámetros concentrados donde cada par de elementos RC modelan un trozo de poro.
Algunos autores han propuesto líneas de transmisión para el modelado de la impedancia de electrodos coplanares. Otros autores han utilizado las líneas de transmisión para modelar el comportamiento de fase constante con la frecuencia (doble capa electroquímica y procesos de difusión).

\section{Agradecimientos}

Los autores desean expresar su agradecimiento a la Comunidad de Madrid por la financiación del Proyecto $\mathrm{N}$ ㅇ. 07N/0043/99.

\section{REFERENCIAS}

[1] J.E.B. Randles, Dis. Faraday Soc., 1 (1947) 11-19.

[2] J. Llopis y F. COlom, en Proc. 8th Meeting CITCE, pp. 414-427, Butterworths, Londres, (1958).

[3] U. Rammelt y G. Reinhard, Electrochim. Acta, 35 (1990) . 1045-1049.

[4] M. CaI y S.-M. PARK, J. Electrochem. Soc., 143 (1996) 3895-3902.

[5] N. Bonanos, B.C.H. Steele, E.P. Butler, W.B. JoHnson, W.L. Worrell, D.D. MaCdonald y M.C.H. MCKUBRE, en Impedance Spectroscopy. Emphasizing Solid Materials and Systems, p. 301, J.R. MACDONALD (Ed.), Wiley, Nueva York, (1987).

[6] R. DE LEVIE, Electrochim. Acta, 9 (1964) 1231-1245.

[7] R. DE LEVIE, Electrochim. Acta, 10 (1965) 113-130.

[8] R. DE LEVIE, en Advances in Electrochemistry and Electrochemical Engineering, Vol. 6, pp. 329-397, Wiley, Nueva York, (1967).

[9] R. DE LEVIE, Electrochim. Acta, 8 (1963) 751-780.

[10] I.D. RaISTRICK, Electrochim. Acta, 35 (1990) 1579-1586.

[11] T. Ohmori, T. Kimura y H. Masuda, J. Electrochem. Soc., 144 (1997) 1286-1288.

[12] J.R. PARK y D.D. MaCdonald, Corros. Sci., 23 (1983) 295-315.

[13] X. Ren y P.G. PicKUP, Electrochim. Acta, 41 (1996) 1877 1882.

[14] D.D. MaCdonald, M. Urquidi-MaCdonald, S.D. BHAKTA y B.G. POUnd, J. Electrochem. Soc., 138 (1991) 1359-1363.

[15] K. Eloot, F. Debuyck, M. Moors y A.P. Van Peteghem, J. Appl. Electrochem., 25 (1995) 326-333.

[16] H. Keiser, K.D. BeCCU y M.A. GuTJAHr, Electrochim. Acta, 21 (1976) 539-543.

[17] J.P. Candy, P. Foullloux, M. Keddam y H. Takenout1, Electrochim. Acta, 26 (1981) 1029-1034.

[18] J.P. Candy, P. Foullloux, M. Keddam y H. Takenouti, Electrochim. Acta, 27 (1982) 1585-1593.

[19] J.M. Bastidas, E.M. Mora y S. Feliu, Werkst. Korros., 41 (1990) 343-347. 
[20] J.M. Bastidas y E.M. Mora, Can. Metall. Quart., 37 (1998) 57-65.

[21] S. Feliu, J.M. Bastidas y M. Morcillo, JOCCA. Surf. Coat. Int., 68 (1985) 133-136.

[22] S. Feliu, J.M. Bastidas y M. Morcillo, Rev. Iberoam. Corros. Prot., 14 (1983) 319-322.

[23] A. Nishikata, Y. Ichihara y T. Tsuru, Corros. Sci., 37 (1995) 897-911.

[24] A. Nishikata, Y. IChihara y T. Tsuru, Electrochim. Acta, 41 (1996) 1057-1062.
[25] S. Feliu, V. Feliu y J.M. Bastidas, Rev. Iberoam. Corros. Prot., 14 (1983) 359-366.

[26] S.H. Liu, Phys. Rev. Lett., 55 (1985) 529-532.

[27] B.B. MANDELbRot, en The Fractal Geometry of Nature, p. 3, W.H. Freeman, Nueva York, (1983).

[28] J.C. Wang y J.B. Bates, Solid State Ionics, 18/19 (1986) 224-228.

[29] J.C. WANG, J. Electrochem. Soc., 134 (1987) 1915-1920. 